\title{
Identification of mycobacterial GarA as a substrate of protein kinase $G$ from $M$. tuberculosis using a KESTREL-based proteome wide approach
}

\author{
Philipp Mueller ${ }^{1}$, Jean Pieters * \\ Biozentrum, University of Basel, Klingelbergstrasse 50, CH 4056 Basel, Switzerland
}

\section{A R T I C L E I N F O}

\section{Article history:}

Received 13 December 2016

Received in revised form 19 February 2017

Accepted 24 February 2017

Available online 27 February 2017

\section{Keywords:}

Serine

Threonine kinase

KESTREL

Mycobacterium tuberculosis

\begin{abstract}
A B S T R A C T
Signal transduction in bacteria is generally mediated via two-component systems. These systems depend on the transfer of a phosphate molecule from a donor to an acceptor by histidine kinases, thereby activating the acceptor to allow downstream signaling/activation. Several bacterial genomes, including the genome of $M$. tuberculosis, were shown to encode eukaryotic-like kinases. To better understand the function of these kinases and the regulatory networks within which they operate, identification of downstream targets is essential. We here present a straightforward approach for the identification of bacterial Ser/Thr-kinase substrates. This approach is based on the KESTREL (Kinase Tracking and Substrate Elucidation) procedure combined with reversed-phase chromatography and two-dimensional gel electrophoresis. Using this method, GarA was identified as one potential substrate for the mycobacterial Ser/Thr-protein kinase G (PknG). These results show that the modified KESTREL approach can be successfully employed for the identification of substrates for bacterial Ser/Thr-kinases.
\end{abstract}

(C) 2017 Elsevier B.V. All rights reserved.

\section{Introduction}

Mycobacterium tuberculosis is one of the world's most successful killers, causing more deaths annually than any other bacterial pathogen. The reason for its pathogenicity lies within the capacity of M. tuberculosis to survive within host organisms, defying innate immune responses (Armstrong and Hart, 1971; Kaufmann et al., 2005; Nguyen and Pieters, 2005). To survive within hosts, mycobacteria are equipped with an array of regulatory mechanisms. However, the signaling networks underlying these survival mechanisms remain largely unknown.

Regulation of bacterial physiology and/or metabolism is usually ensured through the activity of two-component systems (Stock et al., 2000). Interestingly, besides the classical two-components regulatory systems which are normally used in bacteria to regulate signal transduction events, $M$. tuberculosis also expresses several eukaryotic-like serine/threonine kinases (Cole et al., 1998; Leonard et al., 1998; Shi et al., 1998). The genome of M. tuberculosis contains eleven serine/threonine kinases, potentially involved in the regulation of various signal transduction processes (PknA-PknL) (Av-Gay and Everett, 2000; Cole et al., 1998). Nine out of the eleven serine/threonine kinases are predicted to be transmembrane proteins, having their kinase domain exposed in the bacterial cytosol (Av-Gay and Everett, 2000; Nguyen et al., 2005). The function of these transmembrane serine/threonine kinases may be diverse, with proposed functions ranging from determining growth

\footnotetext{
* Corresponding author.

E-mail address: jean.pieters@unibas.ch (J. Pieters).

1 Current address: Boehringer Ingelheim Pharma GmbH \& Co. KG, Ingelheim, Germany.
}

(Sassetti et al., 2003), cell shape (Kang et al., 2005) and virulence (Papavinasasundaram et al., 2005). In addition to the transmembrane kinases, M. tuberculosis produces two soluble kinases, PknG and PknK, of which only PknG is conserved among pathogenic mycobacteria, including Mycobacterium leprae (Cole et al., 2001; Houben et al., 2006). Furthermore, PknG is expressed in M. bovis BCG as well as M. marinum, two species frequently used as model organisms to study the mechanisms of virulence employed by M. tuberculosis (van der Woude et al., 2014; Cambier et al., 2014; Pieters, 2008). Upon infection of macrophages, PknG is readily secreted into the macrophage cytosol, where it blocks phagosome-lysosome fusion thereby allowing the intracellular survival of pathogenic mycobacteria (Nguyen and Pieters, 2006; Scherr et al., 2007b; Walburger et al., 2004).

For most of the mycobacterial serine/threonine kinases, little is known about their physiological substrates and the role they play in bacterial signal transduction and pathogenesis. In order to understand the function of these kinases and to elucidate their role in signal transduction it is essential to identify their substrates. In this paper, we present a straightforward approach for the identification of mycobacterial substrates for serine/threonine kinases. This method is based on the so-called KESTREL (Kinase Tracking and Substrate Elucidation) approach. For KESTREL, cell extracts are separated using ion-exchange chromatography in order to separate endogenous protein kinases from their substrates thereby reducing background phosphorylation and sample complexity (Knebel et al., 2001). Subsequently, the resulting fractions are subjected to phosphorylation using high concentrations of the protein kinase of interest and $\left[\gamma^{32} \mathrm{P}\right]$ ATP of high specific radioactivity (Knebel et al., 2001). Various adaptations have been 
reported for the original KESTREL approach, including a heat treatment step to inactivate endogenous kinases (Troiani et al., 2005), combining it with chemical cleavage to generate a broad range peptide library (Gander et al., 2009) and the introduction of engineered nucleotide analogues (Shah et al., 1997; Muller et al., 2016). However, limitations of such modifications include denaturation of potential substrates due to heat treatment, an inability to recover peptides as well as loss of kinase consensus sites following chemical cleavage and a limited applicability of the usage of engineered nucleotide analogues (Gander et al., 2009; Muller et al., 2016). In addition, KESTREL was originally developed using a eukaryotic system and has since been used to identify kinase substrates in mammalian cells, tissues and eukaryotic parasites (Brandt and Bailey, 2013; Cohen and Knebel, 2006); To the best of our knowledge, KESTREL has not been used for the identification of kinase substrates in prokaryotic cells. Here, we have combined KESTREL with subsequent purification by reversed-phase chromatography and twodimensional gel electrophoresis (2D-IEF/SDS-PAGE) allowing substrate purification based on radiolabeled protein rather than by repeated kinase assays. This combination of proteome fractionation, in vitro kinase assays and reversed-phase chromatography with narrow range two-dimensional gel electrophoresis and MS/MS protein identification is applicable to any cellular system. However, this method is probably most suited to detect putative kinase substrates in lower organisms like bacteria, due to their lower proteomic complexity.

Using this approach, we have identified the forkhead-associated (FHA) domain-containing protein GarA as a mycobacterial substrate for PknG, which has been shown to be able to be phosphorylated in vitro by a variety of mycobacterial kinases, including PknG (O'Hare et al., 2008; Tiwari et al., 2009). These results suggest that the method presented here may provide a powerful tool for the detection of substrates of mycobacterial kinases, in particular, and more generally of prokaryotic signal transduction pathways.

\section{Materials and methods}

\subsection{Cells, plasmids and reagents}

M. bovis BCG $\Delta p k n G$ (strain Pasteur) was propagated in 7H9 mycobacterial medium (Difco) supplemented with $10 \%$ OADC. The $\mathrm{His}_{6}{ }^{-}$ PknG construct and $\mathrm{PknG}_{\mathrm{K} 181 \mathrm{M}}$ have been described and characterized before (Koul et al., 2001; Walburger et al., 2004). [ $\gamma^{32}$ P]ATP and 2D reagents were from Amersham.

\subsection{Expression of $P k n G$ and $P k n G_{K 181 M}$}

For expression and purification of N-terminally $\mathrm{His}_{6}$-tagged PknG and $\mathrm{PknG}_{\mathrm{K} 181 \mathrm{M}}$, Escherichia coli strain BL21 was transformed with pET15-pknG/pknG $G_{K 181 M}$ to obtain $\mathrm{His}_{6}-\mathrm{PknG} / \mathrm{PknG}_{\mathrm{K} 181 \mathrm{M}}$. Transformants were grown in LB medium containing $100 \mu \mathrm{g}$ ampicillin per liter until $\mathrm{OD}_{600}$ reached $0.5-0.6$ and induced with $0.1 \mathrm{mM}$ IPTG at $22{ }^{\circ} \mathrm{C}$ for $16 \mathrm{~h}$ at $160 \mathrm{rpm}$. Cells were harvested by centrifugation ( $3000 \times \mathrm{g}, 15 \mathrm{~min})$, resuspended in $50 \mathrm{mM}$ Tris $(\mathrm{pH} 7.5) / 50 \mathrm{mM} \mathrm{NaCl}, 10 \mathrm{mM}$ imidazol including protease inhibitors (Complete, Roche). Bacteria were homogenized by 3 passages through a French press at 1000 psi (French pressure cell $40 \mathrm{~K}$, type FA-080A-E, Spectronic Instruments, Polytec, Valdbronn, Germany), and the lysates were cleared by centrifugation at 20,000 $\times g$ for $1 \mathrm{~h}$ and subsequent filtration $(0.45 \mu \mathrm{m})$. His-tagged proteins were purified on HisTrap $\mathrm{Ni}^{2+}$-Sepharose chelating and Superdex 200 HiLoad 16/60 columns (Amersham) (Scherr et al., 2007b).

\subsection{Anion exchange chromatography.}

M. bovis BCG Pasteur $\Delta p k n G\left(2000 \mathrm{OD}_{600}\right.$ units) was harvested by centrifugation, resuspended in homogenization buffer (HB; composed of $10 \mathrm{mM}$ triethanolamine, $10 \mathrm{mM}$ acetic acid, $1 \mathrm{mM}$ EDTA, $0.25 \mathrm{M}$ sucrose $\mathrm{pH}$ 7.4) supplemented with protease inhibitors (Complete, EDTA free, Roche) and homogenized as described above.

Homogenates were cleared by centrifugation $\left(1 \mathrm{~h} / 4{ }^{\circ} \mathrm{C} / 100,000 \times \mathrm{g}\right)$ and subsequent filtration $(0.45 \mu \mathrm{m})$. The cleared cytosol was then loaded on a $1 \mathrm{ml}$ MonoQ anion exchange column (Amersham) and eluted with a $20 \mathrm{ml}$ linear salt gradient (buffer A: HB without sucrose; buffer B: HB w/o sucrose and $0.5 \mathrm{M} \mathrm{NaCl}$ ). Fractions were collected, pooled as indicated and assayed for potential PknG substrates as described in Section 2.4 (Scherr et al., 2007a).

\subsection{Kinase assay}

Kinase assays were performed as $200 \mu \mathrm{l}$ reactions by diluting the MonoQ fractions tenfold in PknG reaction buffer $(50 \mathrm{mM}$ Tris/ $\mathrm{HCl}$ $\mathrm{pH} 7.5,5 \mathrm{mM} \mathrm{MnCl}, 1 \mathrm{mM}$ DTT) supplemented with $0.25 \mu \mathrm{Ci}$ $\left[\gamma^{32} \mathrm{P}\right]$ ATP and $0.5 \mu \mathrm{M}$ PknG. Reactions were incubated for $30 \mathrm{~min}$ at $37^{\circ} \mathrm{C}$ in a heating block while shaking at $1000 \mathrm{rpm}$ and terminated by addition of $5 \times$ SDS-sample buffer (Laemmli) and boiling at $95{ }^{\circ} \mathrm{C}$ (for 1DRDS-PAGE (Laemmli, 1970)). Alternatively samples were prepared for the analysis by 2D-IEF/SDS-PAGE, as outlined in the protein analysis and identification section below. For the final protein identification by mass spectrometry, the reactions were also supplemented with $20 \mu \mathrm{M}$ of unlabeled ATP. Reactions were either directly analyzed by 1D-SDSPAGE (12.5\%) and/or 2D-IEF/SDS-PAGE (18/24 cm nl (nonlinear) pH 310 , Amersham) or subjected to further purification and concentration by reversed phase C4 chromatography as described in Section 2.5 .

\subsection{Reversed phase C4 chromatography}

For reversed phase (C4) chromatography samples were acidified with trichloroacetic acid (TFA) to a $1 \%$ final concentration and loaded on C4 columns (GRACEVYDAC BIO-Select Extraction columns Reversed Phase C4 SPE1000 and SPE3000) pre-wetted with 50\% acetonitrile/ $0.1 \%$ TFA and equilibrated with $0.1 \%$ TFA. After washing extensively with $0.1 \%$ TFA columns were eluted with the indicated concentrations of acetonitrile/ $0.1 \%$ TFA. The eluted samples were dried in a speedvac and stored at $-80{ }^{\circ} \mathrm{C}$ until further use.

\subsection{Protein analysis and identification}

Proteins were analyzed using one-dimensional (1-D) (12.5\%) SDSPAGE or two-dimensional (2-D) IEF (IPG/ Amersham)/ SDS-PAGE as described before (Ferrari et al., 1999; Tulp et al., 1994). Samples were dissolved in immobilized pH gradient (IPG) sample buffer (7 M urea, $2 \mathrm{M}$ thiourea, 2\% NP40, 2\% Pharmalyte 3-10, 1\% beta-mercaptoethanol, $2 \%$ final concentration of the IPG buffer corresponding to the $\mathrm{pH}$ range of the IPG strips and $50 \mathrm{mM}$ (dithiothreitol (DTT) and separated according to the manufacturer's protocol. The IPG strips used were $18 / 24 \mathrm{~cm}$ NL pH 3-10 and 18/24 cm NL pH 3-5.6. Gels (1-D SDS-PAGE and 2-D IEF/ SDS-PAGE) were either dried and exposed to autoradiography film (Amersham) or stained with Colloidal blue or Sypro Ruby (Invitrogen) according to the manufacturer's protocol to visualize proteins. Candidate protein spots were cut from the gels and subjected to in-gel trypsin digestion (Perrot et al., 1999). The trypsin digested proteins were analyzed by capillary liquid chromatography tandem MS (LC/MS/MS) using a 300SB C-18 column (0.3x50mm) (Agilent Technologies, Basel, Switzerland) connected on line to an Orbitrap FT hybrid instrument (Thermo Finnigan, San Jose, CA, USA). A linear gradient from 2 to $60 \%$ solvent B $(0.1 \%$ formic acid and $80 \%$ acetonitrile in water $)$ in solvent A ( $0.1 \%$ formic acid and $2 \%$ acetonitrile in water) in 85 min was delivered with a Rheos 2200 pump (Flux Instruments, Basel, Switzerland) at a flow of $50 \mu \mathrm{l} / \mathrm{min}$. A pre-column split was used to reduce the flow to approximately $500 \mathrm{nl} / \mathrm{min}$. Ten $\mu \mathrm{l}$ sample were injected with an autosampler thermostatted to $4{ }^{\circ} \mathrm{C}$ onto a trap column (300SB C-18 column, $0.3 \times 50 \mathrm{~mm}$, Agilent Technologies, Basel, Switzerland) for efficient desalting. The eluting peptides were ionized at $1.6 \mathrm{kV}$. The mass 
spectrometer was operated in a data-dependent fashion so that peptide ions were automatically selected for fragmentation by collision-induced dissociation (MS/MS) in the Orbitrap. The MS/MS spectra were then searched against the data bank using TurboSequest software (Gatlin et al., 2000).

\section{Results}

3.1. Identification of a $20 \mathrm{kDa}$ protein in mycobacterial lysates phosphorylated by PknG

The eukaryotic-like serine/threonine protein kinase $\mathrm{G}(\mathrm{PknG})$ is secreted in the macrophage cytosol where it blocks the fusion of the mycobacterial phagosome with macrophage lysosomes by an as yet uncharacterized mechanism thereby allowing mycobacterial survival (Walburger et al., 2004; Cowley et al., 2004). To investigate the presence of a potential PknG substrate within mycobacteria, crude cytosolic extracts were prepared from Mycobacterium bovis BCG $\Delta$ pknG. The M. bovis BCG $\Delta p k n G$ strain was used in order to avoid phosphorylation of potential substrates by endogenous PknG, which might co-purify with potential substrate(s). Extracts were chromatographed on MonoQ anion exchange columns and eluted with a linear salt gradient (Fig. 1A). Fractions were diluted in PknG reaction buffer and subjected to a kinase assay either in the presence or absence of recombinant PknG to exclude phosphorylation by kinases that might be co-purified from the mycobacterial lysates. Kinase reactions were terminated by

A

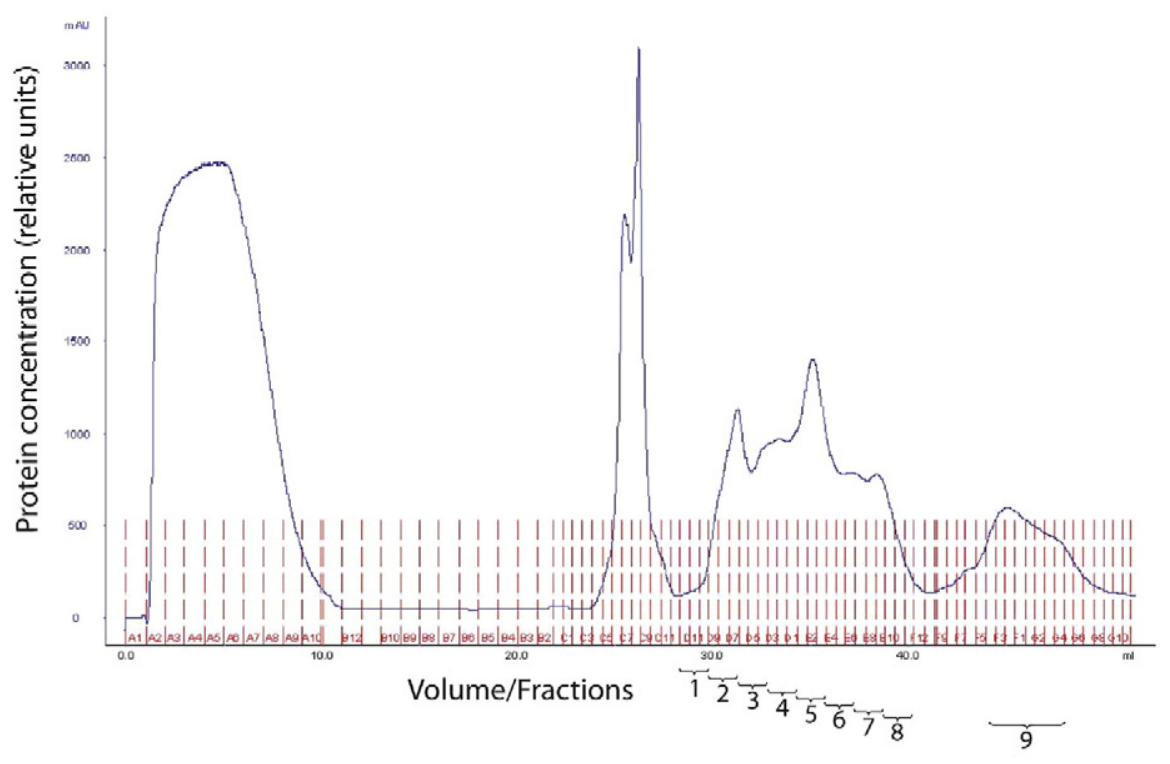

B

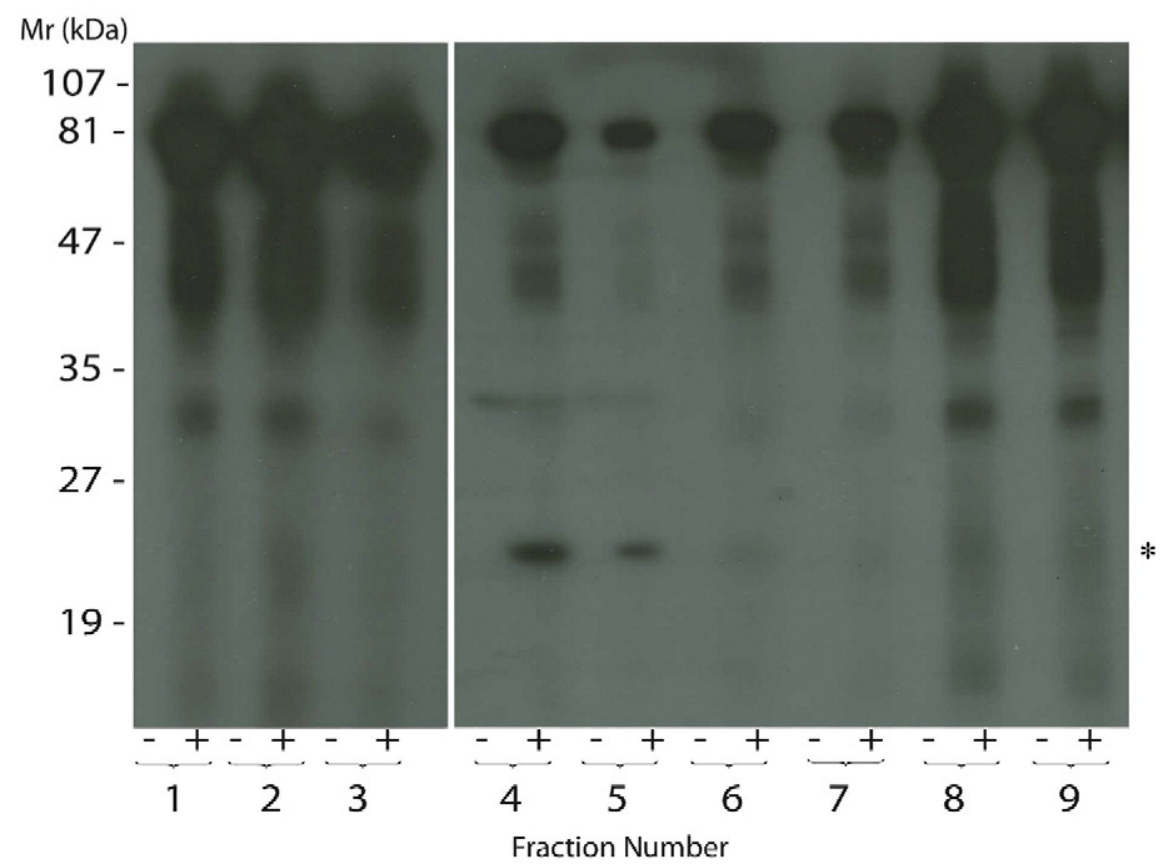

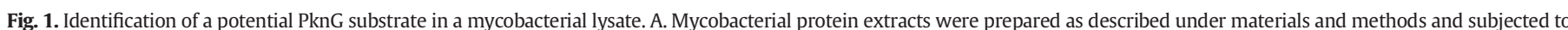

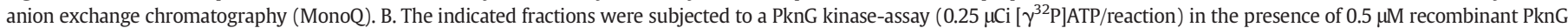

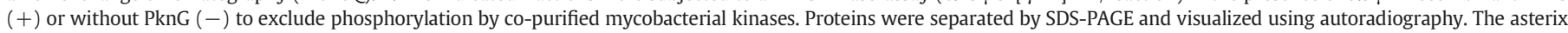
indicates the $20 \mathrm{kDa}$ protein specifically phosphorylated by PknG. 
dilution in Laemmli buffer, and phosphorylated proteins analyzed by $12.5 \%$ SDS-PAGE and autoradiography (Fig. 1B). While the presence of PknG resulted in the appearance of a prominently phosphorylated band at $\sim 80 \mathrm{kD}$, representing autophosphorylated PknG (Scherr et al., 2007a), in fraction 4 and 5, corresponding to a $0.29-0.33 \mathrm{M} \mathrm{NaCl}$ concentration, a protein of apparent molecular mass $20 \mathrm{kDa}$ became phosphorylated only when PknG was present (Fig. 1B, asterix). In order to exclude phosphorylation of this $20 \mathrm{kDa}$ protein by an E. coli kinase that may have been co-purified with recombinant $\mathrm{His}_{6}-\mathrm{PknG}$, the kinase inactive version of $\mathrm{PknG}, \mathrm{His}_{6}-\mathrm{PknG}_{\mathrm{K} 181 \mathrm{M}}$ (Koul et al., 2001; Walburger et al., 2004) expressed in E. coli and purified using exactly the same protocol as used for the purification of wild type PknG was used as a comparison. When $\mathrm{His}_{6}-\mathrm{PknG}_{\mathrm{K} 181 \mathrm{M}}$ was used instead of wild type PknG, no phosphorylation of the $20 \mathrm{kDa}$ protein occurred (Fig. 2A). Together these results suggest that the $20 \mathrm{kDa}$ protein is a mycobacterial protein specifically phosphorylated by PknG.

\subsection{Biochemical characterization of the putative protein kinase $G$ substrate}

To further characterize and purify the $20 \mathrm{kDa}$ protein, fractions containing the $20 \mathrm{kDa}$ putative PknG substrate were pooled, and all subsequent analysis was performed on this material. To determine the isoelectric point of the $20 \mathrm{kDa}$ protein, a PknG kinase reaction performed on the pooled candidate fractions was separated by broad range (NL pH 3-10 18/24 cm) two-dimensional isoelectric focusing SDS-PAGE. The gels were dried and subjected to autoradiography as described above. The $20 \mathrm{kDa}$ candidate protein was found to focus at an acidic pH of $~ 3.5-4.5$ (Fig. 2B).

\subsection{Identification of the putative PknG substrate using reversed-phase} chromatography combined with narrow range two-dimensional gel electrophoresis and MS/MS protein identification

To further reduce the complexity of the pooled candidate fractions and to allow for protein concentration, the PknG kinase reaction containing the $\left[\gamma^{32} \mathrm{P}\right]$ labeled $20 \mathrm{kDa}$ protein was subjected to reversedphase chromatography using a disposable $\mathrm{C} 4$ column. Besides reducing the complexity of the protein sample, in addition to concentrating it, the reversed-phase chromatography step removes all free $\left[\gamma^{32} \mathrm{P}\right]$ ATP resulting in a sample containing exclusively protein bound radioactivity. To follow the purification and keep track of the PknG substrate, a portion of each fraction was neutralized with $1 \mathrm{M}$ Tris/ $\mathrm{HCl} \mathrm{pH} 8$ and subjected to $12.5 \%$ SDS-PAGE and autoradiography (Fig. 3A). The dried $40 \%$ acetonitrile fraction which contained the bulk of the $20 \mathrm{kDa}$ protein was dissolved in 2D sample buffer and subjected to narrow range twodimensional IEF SDS-PAGE (NL pH 3-5.618/24 cm). The gels were fixed and stained with SYPRO Ruby. The spot containing the $\left[\gamma^{32} \mathrm{P}\right]$ labeled $20 \mathrm{kDa}$ protein, indicated by an arrow head (Fig. 3B), was excised from the gel and subjected to in-gel trypsin digestion followed by MS/MS mass spectrometry. The excised protein spot contained a single protein species. MS/MS analysis (sequence coverage 36\%, see Fig. 3C) identified the protein as $M$. bovis BCG GarA.

\section{Conclusions}

While the presence of eukaryotic-like serine/threonine kinases in pathogenic mycobacteria has generated a considerable amount of interest, the signaling pathways controlled by these enzymes remain largely unknown. However, there are no straightforward methods described for the identification and characterization of substrates of serine/ threonine kinases from prokaryotes. In this paper, we describe the usage of a modified KESTEL (Kinase Tracking and Substrate Elucidation) procedure (Knebel et al., 2001) combined with reversed-phase chromatography and two-dimensional gel electrophoresis to elucidate bacterial serine/threonine kinase substrates.

Using this approach we were able to identify the forkhead associated (FHA) domain containing protein GarA, that is widely used as a substrate for mycobacterial kinases and was also shown to interact with PknG (Tiwari et al., 2009; O'Hare et al., 2008; Villarino et al., 2005), as a potential substrate of protein kinase $\mathrm{G}$ from pathogenic mycobacteria.

While the KESTREL approach has been successfully used to identify a number of substrates for eukaryotic kinases (Cohen and Knebel, 2006), its applicability to prokaryotic serine/threonine kinases has not been tested to date. The data presented here validate our approach, which is based on the KESTREL method (Knebel et al., 2001) but is combined with reversed phase chromatography and high resolution narrow range 2-D IEF/SDS-PAGE. The approach that is described here differs from the classical KESTREL method in that we initially employed cell fractionation and chromatography techniques combined with kinase assays, as described for KESTREL, but perform all the subsequent purifications on the already phosphorylated substrate. Such a procedure allows a straight forward monitoring of the purification process and eliminates the need to perform further kinase reactions after every purification step. Inclusion of reversed phase chromatography improved

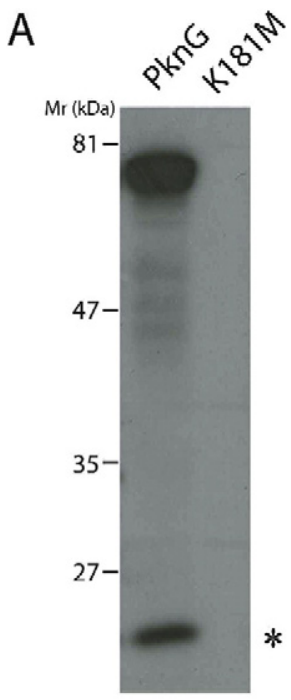

B

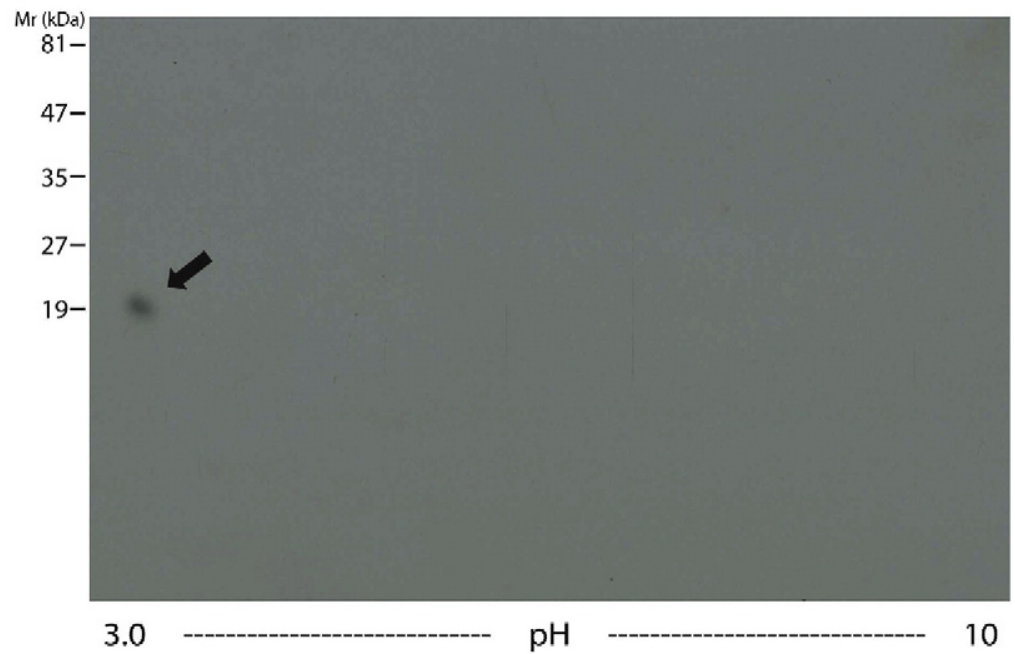

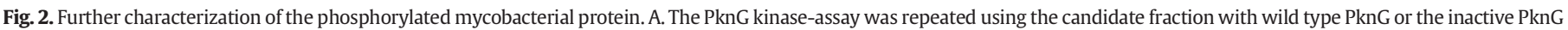
$\left(\mathrm{PknG}^{\mathrm{K} 181 \mathrm{M}}\right)$. The candidate substrate is indicated by an asterix. B. The potential substrate of PknG was analyzed by 2D IEF/SDS-PAGE as described in materials and methods. 


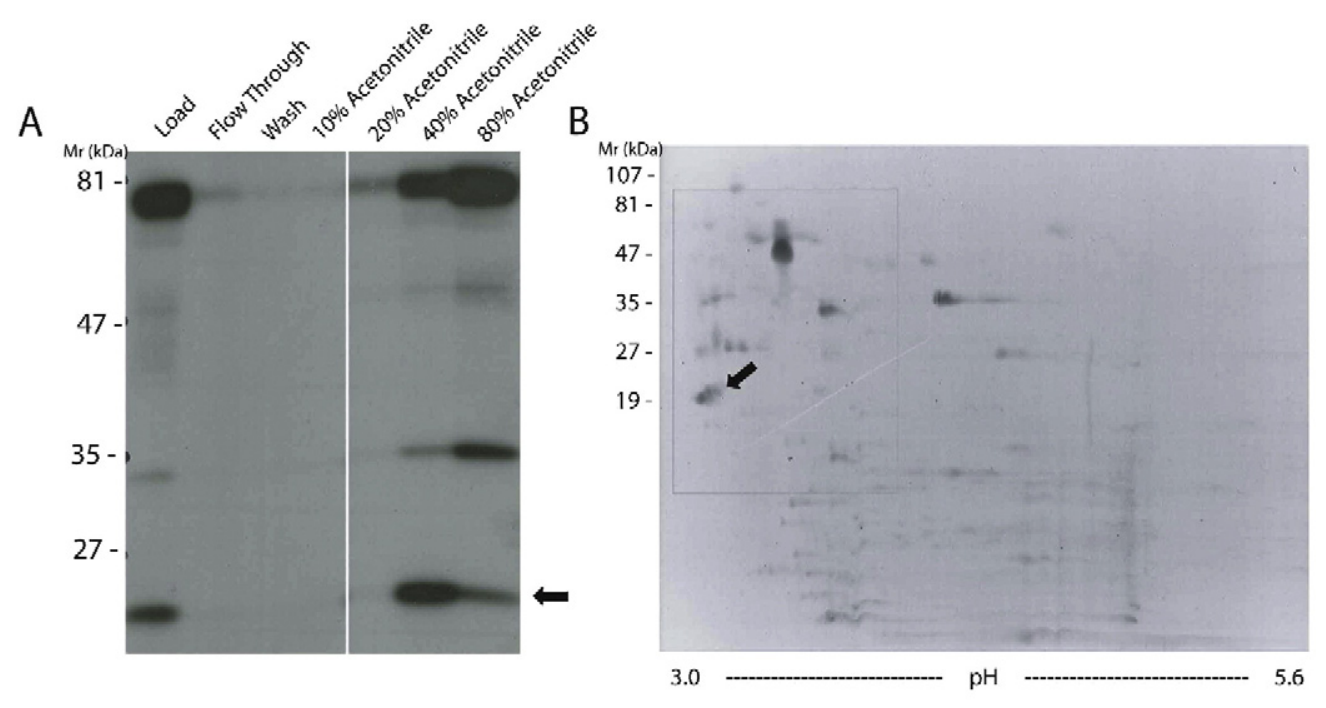

C MTDMNPDIEKDQTSDEVTVETTSVFRADFLSELDAPAQAGTESAVSGVEGLPPGSALLVV 60

KRGPNAGSRFLLDQAITSAGRHPDSDIFLDDVTVSRRHAEFRLENNEFNVVDVGSLNGTY 120

VNREPVDSAVLANGDEVQIGKFRLVFLTGPKQGEDDGSTGGP 162

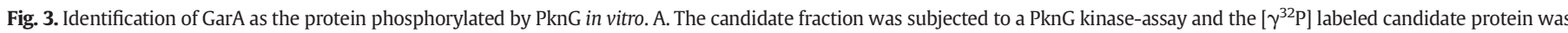

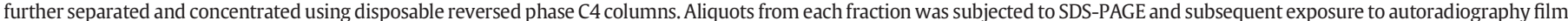

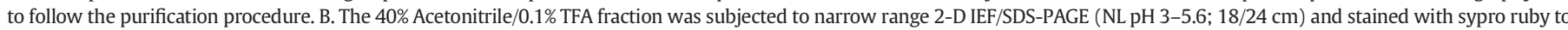

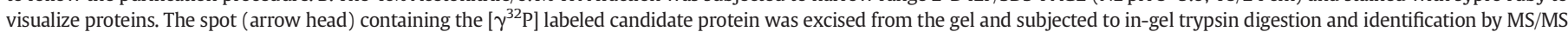

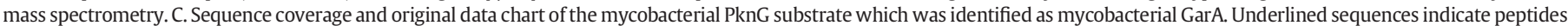
identified by mass spectrometry.

the assay greatly since with a single purification step, besides the benefit of further enrichment and purification of the candidate protein, all nonprotein-bound radioactivity is removed from the samples, making the subsequent handling of the radioactive protein sample safer and omitting the need to perform a new kinase reaction after every purification step.

It should be noted that we have used extracts from Mycobacterium bovis BCG as a source of potential substrates(s), and that the $M$. bovis BCG genome shows several deletions as compared to Mycobacterium tuberculosis therefore potentially excluding substrates that may be selectively expressed in Mycobacterium tuberculosis. Also, whether or not the substrate identified here, GarA, is a physiological substrate of protein kinase $\mathrm{G}$ remains to be determined. Interestingly, a protein with 70.5\% identity to GarA, named OdhI, is phosphorylated by PknG from Corynebacteria (a kinase with $45.2 \%$ identity to PknG from $M$. bovis, LALIGN) (Huang et al., 1990; Niebisch et al., 2006). It is however important to note that GarA, as well as Odh1, contain a forkhead associated (FHA) domain. FHA-domains are phosphoprotein-recognition units (Durocher and Jackson, 2002; Pallen et al., 2002), and notably, in GarA, the FHA-domain spans one third of the full protein length. Together with the fact that all mycobacterial kinases characterized thus far have been shown to undergo autophosphorylation (Boitel et al., 2003; Koul et al., 2001; Young et al., 2003) it is possible that GarA, because of its FHA domain, is a generic substrate for this class of serine/threonine kinases, rather than a physiological substrate within living bacteria. This would be consistent with the finding that various FHA-containing proteins are phosphorylated by mycobacterial kinases, including PknG, PknB, PknD, PknE, PknF and PknH (Grundner et al., 2005; Molle et al., 2003; Molle et al., 2004). On the other hand, phosphorylation of FHA domain containing proteins by mycobacterial serine/threonine kinases could be a key step in regulating signal-transduction pathways through this class of kinases.

The procedure outlined here may allow the identification and classification of additional substrates specific to a given mycobacterial serine/ threonine protein kinase and possibly contribute to a better understanding of the signal transduction pathways regulated by this class of enzymes in mycobacteria.

\section{Acknowledgement}

We thank Lotte Kuhn for expert technical assistance and Paul Jenoe and Suzanne Moes for mass spectrometry as well as members of the Pieters' lab for critical reading. This work was supported by the Swiss National Science Foundation, the World Health Organization, the Olga Mayenfish Stiftung, the UBS Optimus Foundation, the Swiss Life Jubileum Fund and the Canton of Basel.

\section{References}

Armstrong, J.A., Hart, P.D., 1971. Response of cultured macrophages to Mycobacterium tuberculosis, with observations on fusion of lysosomes with phagosomes. J. Exp. Med. $134,713-740$

Av-Gay, Y., Everett, M., 2000. The eukaryotic-like Ser/Thr protein kinases of Mycobacterium tuberculosis. Trends Microbiol. 8, 238-244.

Boitel, B., Ortiz-Lombardia, M., Duran, R., Pompeo, F., Cole, S.T., Cervenansky, C., Alzari, P.M., 2003. PknB kinase activity is regulated by phosphorylation in two Thr residues and dephosphorylation by PstP, the cognate phospho-Ser/Thr phosphatase, in Mycobacterium tuberculosis. Mol. Microbiol. 49, 1493-1508.

Brandt, G.S., Bailey, S., 2013. Dematin, a human erythrocyte cytoskeletal protein, is a substrate for a recombinant FIKK kinase from Plasmodium falciparum. Mol. Biochem. Parasitol. 191, 20-23.

Cambier, C.J., Falkow, S., Ramakrishnan, L., 2014. Host evasion and exploitation schemes of Mycobacterium tuberculosis. Cell 159, 1497-1509. 
Cohen, P., Knebel, A., 2006. KESTREL: a powerful method for identifying the physiological substrates of protein kinases. Biochem. J. 393, 1-6.

Cole, S.T., Brosch, R., Parkhill, J., Garnier, T., Churcher, C., Harris, D., Gordon, S.V., Eiglmeier K., Gas, S., Barry 3rd, C.E., et al., 1998. Deciphering the biology of Mycobacterium tuberculosis from the complete genome sequence. Nature 393, 537-544.

Cole, S.T., Eiglmeier, K., Parkhill, J., James, K.D., Thomson, N.R., Wheeler, P.R., Honore, N., Garnier, T., Churcher, C., Harris, D., et al., 2001. Massive gene decay in the leprosy bacillus. Nature 409, 1007-1011.

Cowley, S., Ko, M., Pick, N., Chow, R., Downing, K.J., Gordhan, B.G., Betts, J.C., Mizrahi, V., Smith, D.A., Stokes, R.W., Av-Gay, Y., 2004. The Mycobacterium tuberculosis protein serine/threonine kinase PknG is linked to cellular glutamate/glutamine levels and is important for growth in vivo. Mol. Microbiol. 52, 1691-1702.

Durocher, D., Jackson, S.P., 2002. The FHA domain. FEBS Lett. 513, 58-66.

Ferrari, G., Langen, H., Naito, M., Pieters, J., 1999. A coat protein on phagosomes involved in the intracellular survival of mycobacteria. Cell 97, 435-447.

Gander, S., Martin, D., Hauri, S., Moes, S., Poletto, G., Pagano, M.A., Marin, O., Meggio, F. Jenoe, P., 2009. A modified KESTREL search reveals a basophilic substrate consensus for the Saccharomyces cerevisiae Npr1 protein kinase. J. Proteome Res. 8, 5305-5316.

Gatlin, C.L., Eng, J.K., Cross, S.T., Detter, J.C., Yates 3rd, J.R., 2000. Automated identification of amino acid sequence variations in proteins by HPLC/microspray tandem mass spectrometry. Anal. Chem. 72, 757-763.

Grundner, C., Gay, L.M., Alber, T., 2005. Mycobacterium tuberculosis serine/threonine kinases PknB, PknD, PknE, and PknF phosphorylate multiple FHA domains. Protein Sci. 14, 1918-1921.

Houben, E.N., Nguyen, L., Pieters, J., 2006. Interaction of pathogenic mycobacteria with the host immune system. Curr. Opin. Microbiol. 9, 76-85.

Huang, X.Q., Hardison, R.C., Miller, W., 1990. A space-efficient algorithm for local similarities. Comput. Appl. Biosci. 6, 373-381.

Kang C.M., Abbott, D.W. Park, S.T, Dascher, C.C., Cantley, L.C., Husson, R.N., 2005. The Mycobacterium tuberculosis serine/threonine kinases PknA and PknB: substrate identification and regulation of cell shape. Genes Dev. 19, 1692-1704.

Kaufmann, S.H., Cole, S.T., Mizrahi, V., Rubin, E., Nathan, C., 2005. Mycobacterium tuberculosis and the host response. J. Exp. Med. 201, 1693-1697.

Knebel, A., Morrice, N., Cohen, P., 2001. A novel method to identify protein kinase substrates: eEF2 kinase is phosphorylated and inhibited by SAPK4/p38delta. EMBO J. 20, 4360-4369.

Koul, A., Choidas, A., Tyagi, A.K., Drlica, K., Singh, Y., Ullrich, A., 2001. Serine/threonine protein kinases PknF and PknG of Mycobacterium tuberculosis: characterization and localization. Microbiology 147, 2307-2314.

Laemmli, U.K., 1970. Cleavage of structural proteins during the assembly of the head of bacteriophage T4. Nature 227, 680-685.

Leonard, C.J., Aravind, L., Koonin, E.V., 1998. Novel families of putative protein kinases in bacteria and archaea: evolution of the "eukaryotic" protein kinase superfamily. Genet. Res. 8, 1038-1047.

Molle, V., Girard-Blanc, C., Kremer, L., Doublet, P., Cozzone, A.J., Prost, J.-F., 2003. Protein PknE, a novel transmembrane eukaryotic-like serine/threonine kinase from Mycobacterium tuberculosis. Biochem. Biophys. Res. Commun. 308, 820-825.

Molle, V., Soulat, D., Jault, J.-M., Grangeasse, C., Cozzone, A.J., Prost, J.-F., 2004. Two FHA domains on an $\mathrm{ABC}$ transporter, Rv1747, mediate its phosphorylation by PknF, a Ser/Thr protein kinase from Mycobacterium tuberculosis. FEMS Microbiol. Lett. 234, 215-223.

Muller, A.C., Giambruno, R., Weisser, J., Majek, P., Hofer, A., Bigenzahn, J.W., Superti-Furga G., Jessen, H.J., Bennett, K.L., 2016. Identifying kinase substrates via a heavy ATP kinase assay and quantitative mass spectrometry. Sci. Report. 6, 28107.

Nguyen, L., Pieters, J., 2005. The Trojan horse: survival tactics of pathogenic mycobacteria in macrophages. Trends Cell Biol. 15, 269-276.

Nguyen, L., Pieters, J., 2006. Battle for survival: interplay between pathogenic mycobacteria and the host immune system. Discov. Med. 6, 82-86.

Nguyen, L., Walburger, A., Houben, E., Koul, A., Muller, S., Morbitzer, M., Klebl, B., Ferrari, G., Pieters, J., 2005. Role of protein kinase $G$ in growth and glutamine metabolism of Mycobacterium bovis BCG. J. Bacteriol. 187, 5852-5856.
Niebisch, A., Kabus, A., Schultz, C., Weil, B., Bott, M., 2006. Corynebacterial protein kinase $\mathrm{G}$ controls 2-oxoglutarate dehydrogenase activity via the phosphorylation status of the OdhI protein. J. Biol. Chem. 281, 12300-12307.

O'Hare, H.M., Durán, R., Cerveñansky, C., Bellinzoni, M., Wehenkel, A.M., Pritsch, O., Obal, G., Baumgartner, J., Vialaret, J., Johnsson, K., Alzari, P.M., 2008. Regulation of glutamate metabolism by protein kinases in mycobacteria. Mol. Micobiol. 70, 1408-1423.

Pallen, M., Chaudhuri, R., Khan, A., 2002. Bacterial FHA domains: neglected players in the phospho-threonine signalling game? Trends Microbiol. 10, 556-563.

Papavinasasundaram, K.G., Chan, B., Chung, J.-H., Colston, M.J., Davis, E.O., Av-Gay, Y., 2005. Deletion of the Mycobacterium tuberculosis pknH Gene confers a higher bacillary load during the chronic phase of infection in BALB/c mice. J. Bacteriol. 187, 5751-5760.

Perrot, M., Sagliocco, F., Mini, T., Monribot, C., Schneider, U., Shevchenko, A., Mann, M., Jeno, P., Boucherie, H., 1999. Two-dimensional gel protein database of Saccharomyces cerevisiae (update 1999). Electrophoresis 20, 2280-2298.

Pieters, J., 2008. Mycobacterium tuberculosis and the macrophage: maintaining a balance. Cell Host Microbe 3, 399-407.

Sassetti, C.M., Boyd, D.H., Rubin, E.J., 2003. Genes required for mycobacterial growth defined by high density mutagenesis. Mol. Microbiol. 48, 77-84.

Scherr, N., Honnappa, S., Kunz, G., Mueller, P., Jayachandran, R., Winkler, F., Pieters, J., Steinmetz, M.O., 2007a. From the cover: structural basis for the specific inhibition of protein kinase G, a virulence factor of Mycobacterium tuberculosis. Proc. Natl. Acad. Sci. U. S. A. 104, 12151-12156.

Scherr, N., Honnappa, S., Kunz, G., Mueller, P., Jayachandran, R., Winkler, F., Pieters, J., Steinmetz, M.O., 2007b. Structural basis for the specific inhibition of protein kinase G, a virulence factor of Mycobacterium tuberculosis. Proc. Natl. Acad. Sci. U. S. A. 104, 12151-12156.

Shah, K., Liu, Y., Deirmengian, C., Shokat, K.M., 1997. Engineering unnatural nucleotide specificity for Rous sarcoma virus tyrosine kinase to uniquely label its direct substrates. Proc. Natl. Acad. Sci. U. S. A. 94, 3565-3570.

Shi, L., Potts, M., Kennelly, P.J., 1998. The serine, threonine, and/or tyrosine-specific protein kinases and protein phosphatases of prokaryotic organisms: a family portrait. FEMS Microbiol. Rev. 22, 229-253.

Stock, A.M., Robinson, V.L., Goudreau, P.N., 2000. Two-component signal transduction. Annu. Rev. Biochem. 69, 183-215.

Tiwari, D. Singh, R.K., Goswami, K., Verma, S.K., Prakash, B., Nandicoori, V.K., 2009. Key residues in Mycobacterium tuberculosis protein kinase $\mathrm{G}$ play a role in regulating kinase activity and survival in the host. J. Biol. Chem. 284, 27467-27479.

Troiani, S., Uggeri, M., Moll, J., Isacchi, A., Kalisz, H.M., Rusconi, L., Valsasina, B., 2005. Searching for biomarkers of Aurora-A kinase activity: identification of in vitro substrates through a modified KESTREL approach. J. Proteome Res. 4, 1296-1303.

Tulp, A., Verwoerd, D., Dobberstein, B., Ploegh, H.L., Pieters, J., 1994. Isolation and characterization of the intracellular MHC class II compartment. Nature 369, 120-126.

Villarino, A., Duran, R., Wehenkel, A., Fernandez, P., England, P., Brodin, P., Cole, S.T., Zimny-Arndt, U., Jungblut, P.R., Cervenansky, C., et al., 2005. Proteomic identification of M. tuberculosis protein kinase substrates: PknB recruits GarA, a FHA domain-containing protein, through activation loop-mediated interactions. J. Mol. Biol. 350, 953-963.

Walburger, A., Koul, A., Ferrari, G., Nguyen, L., Prescianotto-Baschong, C., Huygen, K., Klebl, B., Thompson, C., Bacher, G., Pieters, J., 2004. Protein kinase G from pathogenic mycobacteria promotes survival within macrophages. Science 304, 1800-1804.

van der Woude, A.D., Stoop, E.J., Stiess, M., Wang, S., Ummels, R., van Stempvoort, G., Piersma, S.R., Cascioferro, A., Jimenez, C.R., Houben, E.N., et al., 2014. Analysis of SecA2-dependent substrates in Mycobacterium marinum identifies protein kinase G (PknG) as a virulence effector. Cell. Microbiol. 16, 280-295.

Young, T.A., Delagoutte, B., Endrizzi, J.A., Falick, A.M., Alber, T., 2003. Structure of Mycobacterium tuberculosis PknB supports a universal activation mechanism for Ser/Thr protein kinases. Nat. Struct. Biol. 10, 168-174. 